\title{
Multiple features and classifiers for vein based biometric recognition.
}

\author{
Bharathi Subramaniam*, Sudhakar Radhakrishnan \\ Department of Electronics and Communication Engineering, Dr. Mahalingam College of Engineering and Technology, \\ Pollachi 642 003, Tamil Nadu, India
}

\begin{abstract}
An effective fusion scheme is necessary for combining features from multiple biometric traits. This paper presents a method of fusion using multiple features from hand vein biometric traits for Multimodal biometric recognition. In the proposed method, a biometric authentication system using three different set of veins images, such as, finger vein, palm vein and dorsal vein is developed. Here the multiple features from the input vein images are extracted by applying Radon transform, HilbertHuang transform and Dual tree complex wavelet transform for each of the vein images. Once the features are extracted, a feature level fusion is carried out using the optimization algorithm called, Group Search Optimization. Then, recognition is done using the trained features by different classifiers such as support vector machine, fuzzy, neural network, bayes classifier and k-nearest neighbor classifiers. This approach is tested on the standard data bases of finger vein, palm vein and dorsal vein images of the hand. The proposed method provides higher accuracy and lower equal error rate which shows the efficiency of the technique compared with the other existing techniques.
\end{abstract}

Keywords: Finger vein, Palm vein, Dorsal hand vein, Multiple features, Feature level fusion, Multi-modal biometric recognition.

Accepted on February 01, 2017

\section{Introduction}

An organization, like school, college, hospitals, company and industries, there is a need of biometric identification for security and monitoring purpose. Biometric technology used for identification of individual persons by means of their physiological characteristics such as, face, fingerprint, vein, iris, voice, palm print etc., and behavioral traits like keystroke dynamics, gait and signature [1]. The traditional authentication methods have numerous disadvantages, which can be overcome by biometrics method. Biometric information cannot be observed directly, it is difficult to share and recover, there is no necessity to memorize long password. Every biometric technology has its advantages and disadvantages, because no one can satisfy the necessities of all authentication and recognition applications.

For a larger user application, the unimodal biometric system [2] performance is not being enough for the identification. This drawback can be overcome by multimodal biometric systems $[3,4]$, which is a combination of two or more biometric information for the authentication system. The multimodal biometric system improves the recognition rate, accuracy, security and universality [5,6]. In multimodal biometric recognition systems, the fusion [7] of various traits can be carried out at different levels, such as sensor level, feature level [8,9], matching score level [10-12] and decision level [13]. Among different fusion methods, the feature level fusion discriminates features from various modalities and is conserved more than other fusion levels because it contains rich information of biometric traits. The multimodal fusion [14] performed at the feature level is difficult to provide required result because of potential inappropriateness of feature spaces produced by different modalities. The frustration of dimensionality for eternity is an undesirable occurrence in the feature-level fusion process $[15,16]$. The traditional feature level fusion methods like serial feature fusion and parallel feature fusion 16 are not applicable in many applications. With the purpose of fusing different biometric information in feature level fusion with realistically and efficiently, more methods should be developed for exploiting biometric features with balancing and creating appropriate rules for feature level fusion. Feature selection strategies often are applied to explore the effect of irrelevant features on the performance of classifier systems [17]. In this phase, an optimal subset of features which are necessary and sufficient for solving a problem is selected. Feature selection improves the accuracy of algorithms by reducing the dimensionality and removing irrelevant features [18].

Here, multiple features and classifiers with feature level fusion of vein modalities for recognition are proposed. The motivation of this work is choosing of right modality to provide live experience and also conserve the individuality. The selection of two or more images in multimodal biometric system and fusion of their features should be carried out to 
improve the accuracy and to reduce the error. In order to improve the accuracy and to minimize the error rate multiple features and classifiers with feature level fusion of vein modalities for recognition are proposed here. In this method the biometric information is obtained from three different sets of vein images, such as, finger vein, dorsal hand vein and palm vein are used for recognition. The significant features of the three vein images are obtained by using three different transforms, such as, Radon transform [19], Hilbert-Huang transform (kind of empirical mode decomposition) [20,21], and Dual tree complex wavelet transform (DT-CWT) [22]. Here each of the vein images are individually transformed using Radon transform, Hilbert-Huang transform and Dual tree complex wavelet transforms. Therefore, for a single image, three different feature sets are obtained with different sizes and they are resized to the same size. These extracted features are fused at the feature level itself. In this method, the feature level fusion is achieved by generating appropriate rules with the help of optimization algorithm, namely group search optimization (GSO) [23] and then these features are used for recognition. Here five classifiers are used, such as, SVM, fuzzy, neural network, Naive Bayes classifier, K-NN classifier, which is well trained by the optimized feature obtained by GSO and their results are compared. The rest of the paper is organized as follows: The proposed method of optimal feature level fusion for vein modalities is presented in section 2. The detailed experimental results and discussion are given in section 3 and the conclusion is summed up in section 4 .

\section{Experimental Method}

The block diagram of the proposed method is given in the Figure 1. In this method three vein images such as finger vein, dorsal hand vein and palm vein are considered for authentication. Here, after preprocessing, the significant features are extracted from the preprocessed image by using three different transforms such as Hilbert-Hung transform, Radon transform and Dual tree wavelet transform. Then, these extracted features of the different vein images are fused by using an optimal formula generated by the GSO algorithm. Finally, the fused features are given to the different classifiers for training and testing. If the testing result is not accurate then this process is repeated until to get the accurate result.

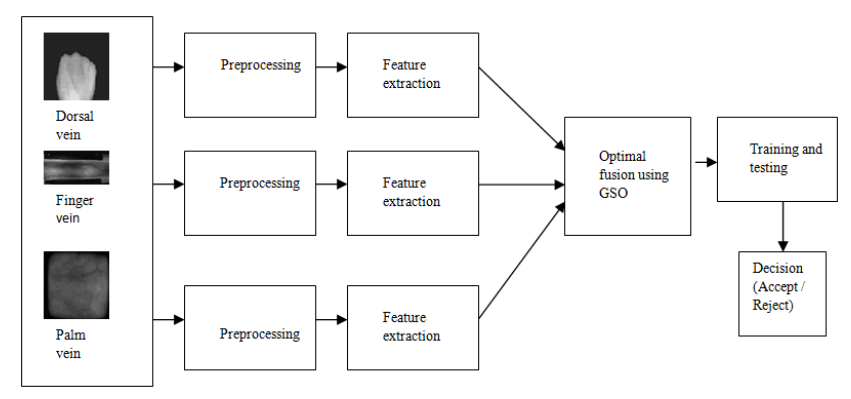

Figure 1. Block diagram of proposed method.

\section{Hilbert-Huang transform}

By employing Empirical Mode Decomposition (EMD) the features of vein images are extracted. Huang [20] established a technique that decomposes a data into a sum of components, each with slowly varying amplitude and phase. Every component of the EMD is called an Intrinsic Mode Function (IMF).

More specifically, the input matrix is converted into vector and the input data is represented by $D(x)$, (Equation 1) and then the application of EMD generates a set of $N$, IMFs $\left\{I M F_{\mathrm{i}}(x)\right\}_{\mathrm{i}=1} \mathrm{~N}$, such that

$D(x)=\sum_{i=0}^{N} I M F_{i}+R(x) \rightarrow(1)$

Where, $R(x)$ is a residual monotonic function

Upon obtaining an IMF, the same procedure is applied to the residual data $R(x)=D^{\prime}(x)-I M F(x)$ to extract the next IMF. From the element with high frequency the IMFs are successively obtained. Hence, the residual data $R(x)$ has the lowest frequency.

\section{Radon transform}

The radon function computes the line integrals from multiple sources along parallel paths, or beams, in a certain direction. To represent a vein image, the radon function takes multiple, parallel-beam projections of the image from different angles by rotating the source around the center of the image. Applying the Radon transform on a vein image $f(x, y)$ for a set of angles can be thought of as computing the projection of the image along the given angles. The resulting projection is the sum of the intensities of the pixels in each direction.

The radon transform can be represented as (Equation 2), by defining,

$$
\begin{aligned}
& \alpha=x \cos \phi+y \sin \phi \\
& R T(\alpha, \varphi)=\int_{-\infty}^{\infty} \int_{-\infty}^{\infty} f(x, y) \delta(\alpha-x \cos \varphi-y \sin \varphi) d x d y \rightarrow(2)
\end{aligned}
$$

Where, $\delta$ is the Dirac delta function. The collection of the RT $(\alpha, \phi)$ at all $\phi$ is the Radon transform of vein image $f(x, y)$.

\section{Dual tree complex wavelet transform (DT-CWT)}

The feature extraction utilizes the multi-level coefficients of decomposition parts of vein images via DT-CWT, which is implemented using a dual-tree structure. The shift invariant and directionality selective properties of the DT-CWT are utilized for extracting features from vein images. Each vein images are decomposed by DT-CWT and the vein feature matrix consists of high frequency decomposition coefficients as well as real and imaginary part from the highest level. 
The vein image features obtained from these three transforms are in different sizes and these feature matrices are resized into the same size feature matrix.

\section{Optimal feature level fusion}

In order to achieve the optimal feature level fusion, the nine set of features obtained from three different vein images are fused using an optimization algorithm called GSO. In GSO, the random population of size $20 \times 17$ is selected considered, the values from one to nine columns are assigned as a parameter to multiply by the corresponding feature matrix of the vein image and the values from ten to seventeen are assigned to select the operators which are already assigned within the range $(0,1)$. These pre-assigned operators are used to perform arithmetic operations between the feature matrices. By these calculations, a single matrix is obtained and is then given to different classifiers for testing and training. If the error rate is higher than predetermined error rate, then the GSO once again generate another $20 \times 17$ vector. This optimization operation is repeated until the error rate is lesser than predetermined error rate.

Group search optimization: Group search optimization algorithm is inspired by animal searching behavior. It may be described as an active moment by which an animal moves to find resources such as food and shelter. Here, the population is referred as a group and all individual present in the group is named as members. There are three types of members present in the group: producers, scroungers and rangers. Producers and scroungers behaviors are based on PS model, and the member rangers perform a random walk motion. The procedure for optimization is given below.

Step 1: Initialize the search solution and head angle

\section{Step 2: Calculate the fitness}

The parameters and operators value generated by GSO are used to fuse the feature matrices from each transform into a single matrix. Then it is trained and tested by using five classifiers and the obtained values from each classifier are sorted and the minimum value is chosen for error rate checking. If the rate is below the specified value, the GSO process is stopped otherwise it generates a new solution. The formula used to find the fitness is given in the Equation 3.

Fitness $=1-\left[\frac{T P+T N}{T P+T N+F P+F N}\right] \rightarrow(3)$

where, TP - true positive, TN - true negative, FP - false positive and FN - false negative.

Step 3: Find the producer of the group by changing the head angle and by analyzing the performance of Scrounger and Ranger.

At the end of this procedure, a new solution is generated. The solution which gives minimum error rate for each classifier is chosen as the best solution.

\section{Results and Discussion}

The experimental results of the proposed method of multiple features and classifiers with feature level fusion for biometric recognition are discussed here. The evaluation metrics employed here are accuracy, FAR (False Acceptance Rate) and FRR (False Rejection Rate). The database utilized for our experimentation is taken from the standard data bases [24-26] for dorsal hand vein, palm vein and finger vein images. The input vein images and their corresponding transformed images are shown in Figure 2.

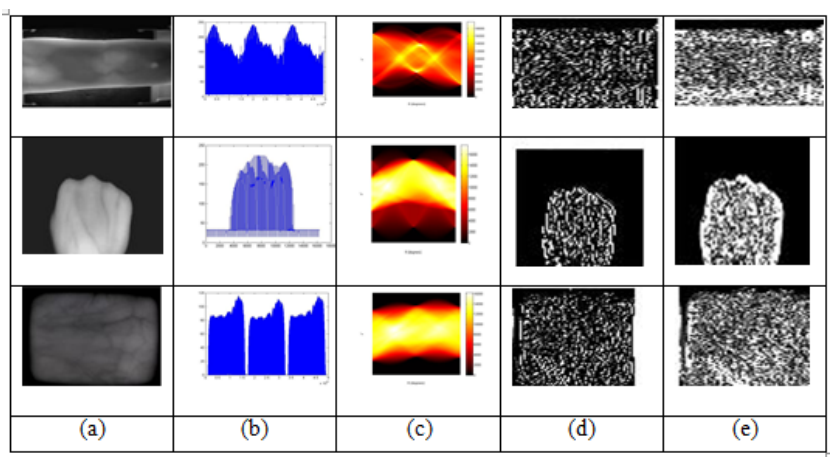

Figure 2. (a) Input vein images (b) output emd of HHT (c) Radon transformed output (d) Real part of DT-CWT (e) Imaginary part of DT-CWT.

The performance analysis is made with the use of evaluation metrics of FAR, FRR and accuracy. These analyses of hand vein images is done using different classifiers such as SVM, fuzzy, neural network, Naive Bayes classifier and K-NN classifier at various threshold levels with the GSO population size is $20 \times 17$ and $10 \times 17$. The plot of FAR, FRR and accuracy are shown in Figures 3-5.
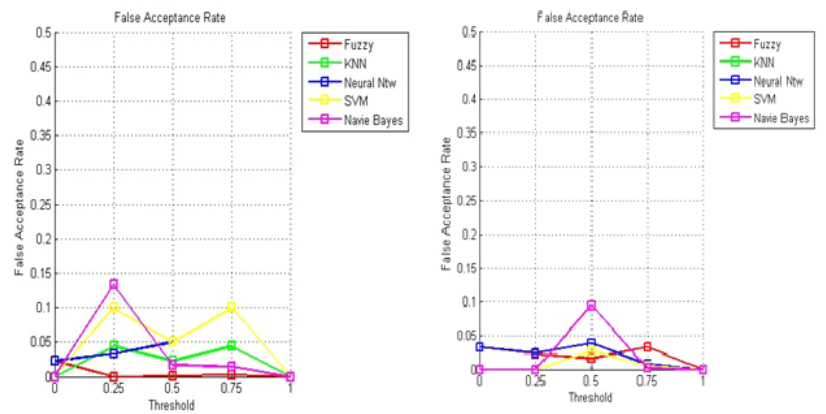

Figure 3. Plot of FAR for (a) population size $10 \times 17$ (b) population size $20 \times 17$.

From these plots we observed that by combining three vein biometric traits the FAR and FRR are low. Among all five classifiers the Fuzzy system provides the lowest rate of zero and also as the population size increases the FAR and FRR decreases. Regarding accuracy, we have obtained higher value irrespective of classifiers. For all types of classifiers we got the accuracy as above $90 \%$ except Navie bayes classifier which provides the accuracy of around $80 \%$. Here as the population size increases the accuracy also increases. The receiver operating characteristic (ROC) curve shown in Figure 6, 
represents the accomplishment and efficiency of the different classifier system. From the ROC curves, we can see that our proposed technique has achieved lower equal error rate (EER) indicating the effectiveness of the proposed technique. The EER for different classifiers with population size of $10 \times 17$ and $20 \times 17$ are given in the Table 1 .

Table 1. Equal error rate for different classifiers.

\begin{tabular}{lll}
\cline { 2 - 3 } & $20 \times 17$ & $10 \times 17$ \\
\hline Kuzzy & 0.017 & 0.014 \\
\hline Neural network & 0.019 & 0.024 \\
\hline SVM & 0.05 & 0.028 \\
\hline Naive Bayes & 0.035 & 0.024 \\
\hline
\end{tabular}

\section{Classifiers}

Population size

Table 2. Comparative Analysis of related work on Multimodal biometric authentication [27-33].

\begin{tabular}{|c|c|c|c|c|}
\hline System & Feature & Level fusion & Methodology & Performance \\
\hline Conti et al. & Iris and fingerprint & Template level & Hamming distance & $\mathrm{FAR}=0, \mathrm{FRR}=5.7 \% \mathrm{EER}=2.36$ \\
\hline \multirow[t]{2}{*}{ Khalifa et al. } & Signature+ Handwriting & Decision level & Logical AND,OR & $E E R=3.80,8.05$ \\
\hline & Signature+ Handwriting & Score level & Arithmetic Average, SVM & $E E R=2.65,3.2$ \\
\hline \multirow[t]{2}{*}{ Lin et al. } & Palm-dorsa vein & Feature level & Multi resolution analysis and & $\mathrm{FAR}=1.5, \mathrm{FRR}=3.5$ \\
\hline & & & Hierarchical integrating function & $E E R=3.75$ \\
\hline \multirow[t]{3}{*}{ Amayeh et al. } & Palm + finger & Feature level & PCA & $E E R=0.523$ \\
\hline & & score level & weighted sum and SVM & $E E R=0.052,0.136$ \\
\hline & & decision level & majority voting scheme & $E E R=0.044$ \\
\hline \multirow[t]{2}{*}{ Kumar and Prathyusha } & Hand vein and knuckle shape & Score level & Matching vein triangulation and & $F A R=1.14$ \\
\hline & & & shape features & $\mathrm{FRR}=1.14$ \\
\hline \multirow[t]{2}{*}{ Raghavendra et al. } & Hand vein and palm print & score level & $\begin{array}{l}\text { Log Gabor transform, weighted sum } \\
\text { rule }\end{array}$ & $\mathrm{FAR}=7.4, \mathrm{FRR}=4.8, \mathrm{EER}=6.1$ \\
\hline & & & $\begin{array}{l}\text { Non standard mask, weighted sum } \\
\text { rule }\end{array}$ & $F A R=2.8, F R R=1.4, E E R=2.2$ \\
\hline Ferrer et al. & $\begin{array}{l}\text { Hand geometry, palm and } \\
\text { finger textures, dorsal hand } \\
\text { vein }\end{array}$ & $\begin{array}{l}\text { Score level and decision } \\
\text { level }\end{array}$ & Simple Sum rule & $F A R=0.01, F R R=0.01, E E R=0.01$ \\
\hline Proposed Method & $\begin{array}{l}\text { Finger vein, Palm vein and } \\
\text { Dorsal vein }\end{array}$ & Feature level & $\begin{array}{l}\text { GSO, Fuzzy, SVM, } \\
\text { K-NN }\end{array}$ & $\begin{array}{l}F A R=0.01 \% \\
E E R=0.014\end{array}$ \\
\hline
\end{tabular}
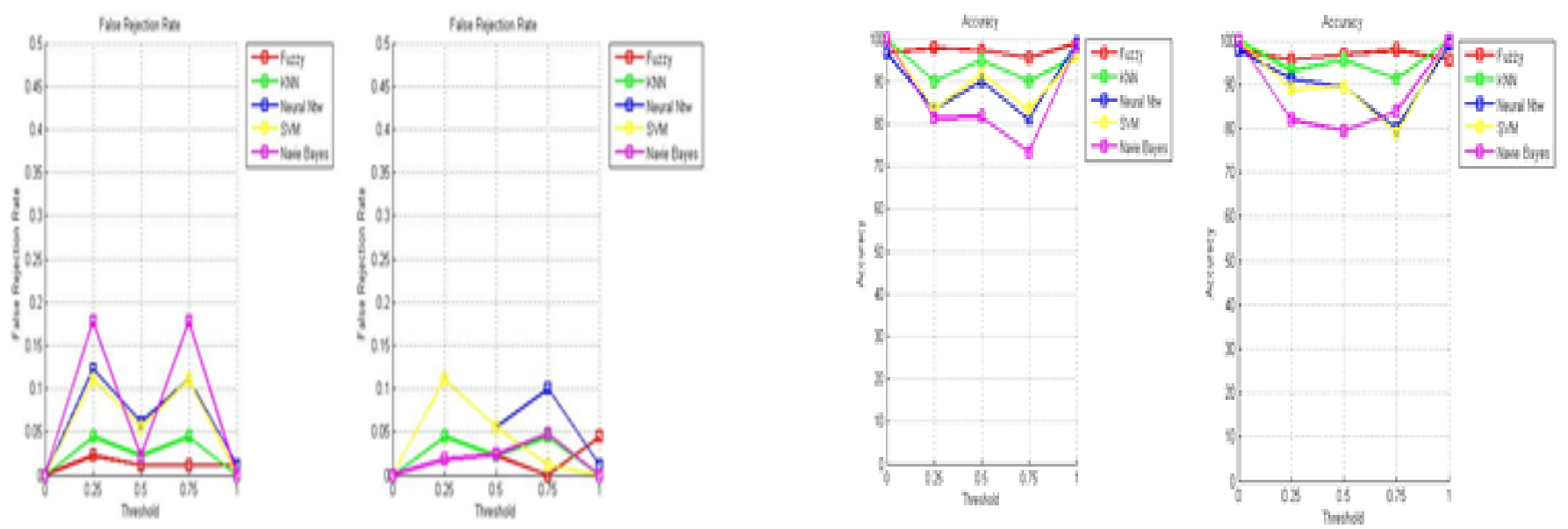

Figure 4. Plot of FRR for (a) population size $10 \times 17$; (b) population size $20 \times 17$.
Figure 5. Plot of Accuracy for (a) population size $10 \times 17$; (b) population size $20 \times 17$. 

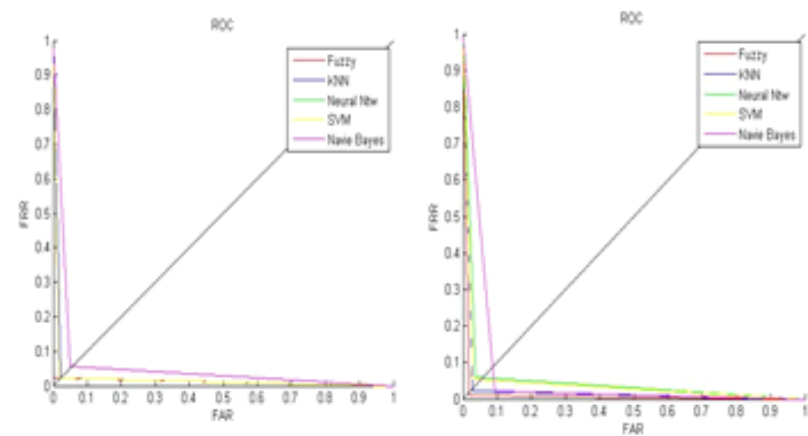

Figure 6. Plot of ROC (a) GSO population size of 20 17; (b) GSO population size of $10 \times 17$.

The detailed comparative analysis of the Multimodal biometric authentication system with their fusion methods is given in Table 2. All the results presented in this table are in terms of EER, FAR and FRR. Here EER is defined as a point at which FAR is equal to FRR. Lower the values of EER, better the performance of the system but it varies according to the imaging techniques, type biometric traits, fusion strategies and number of users in the data base.

\section{Conclusion}

The main significance of the proposed Multimodal biometric system is to improve the performance of the system by fusing multiple features from each of the vein images of hand. Here, dorsal hand vein, palm vein and finger vein images of the hand are considered. The significant features of the input vein images were obtained by applying all the three transforms (Radon transform, Hilbert-Huang transform and Dual tree complex wavelet transform) to each of the vein images. The extracted features are fused by optimal feature level fusion using GSO algorithm. The authentication is done by using the trained features from different classifiers like fuzzy, k-NN, neural network, SVM and naive bayes. The experimental results illustrate that the optimal feature level fusion method for hand vein biometric traits renders fairly good performance compared to traditional methods. The proposed fusion method yields FAR of $0.011 \%$, FAR of $0.031 \%$, EER of $0.014 \%$, which is a remarkable improvement than existing methods.

\section{References}

1. Ross A, Jain AK. Multimodal biometrics: an overview. Proc of 12th European Signal Process. Conf. (EUSIPCO), Vienna, Austria, September 2004.

2. Bar-Cohen Y. Biologically Inspired Intelligent Robots Using Artificial Muscles. Strain 2005; 41: 19-24.

3. Yang W, Huang X, Zhou F, Liao Q. Comparative competitive coding for personal identification by using finger vein and finger dorsal texture fusion. Int J Inf Sci 2014; 268: 20-32.

4. Zhu L, Zhang S. Multimodal biometric identification system based on finger geometry, knuckle print and palm print. Pattern Recogn Lett 2010; 31: 1641-1649.
5. Kanhangad V, Kumar A, Zhang D. A unified framework for contact less hand verification. IEEE Transact Inf Forens Secur 2011; 6: 1014-1027.

6. Lee EC, Jung H, Kim D. New finger biometric method using near infrared imaging. Basel 2011; 11: 2319-2333.

7. Ross A, Jain A. Information fusion in biometrics. Pattern Recogn Lett 2003; 24: 2115-2125.

8. Chin YJ, Ong TS, Teoh ABJ, Goh KOM. Integrated biometrics template protection technique based on fingerprint and palm print feature-level fusion. J Inf Fusion 2014; 18: 161-174.

9. Yang J, Zhang X. Feature-level fusion of fingerprint and finger-vein for personal identification. Pattern Recogn Lett 2012; 33: 623-628.

10. Sakthivel K, Jayanthiladevi A, Kavitha C. Automatic detection of lung cancer nodules by employing intelligent fuzzy cmeans and support vector machine. Biomed $\mathrm{Re}$ 2016; 27: S123-S127.

11. Hanmandlu M, Grover J, Madasu VK, Vasirkala S. Score level fusion of hand based biometrics using t-norms. IEEE Int Conf Technol Home Land Security, Waltham, 2010, IEEE.

12. Bharathi S, Sudhakar R, Balas VE. Biometric Recognition Using Fuzzy Score Level Fusion. Int J Adv Intell Paradigms 2014; 6: 81-94.

13. Saleh IA, Alzoubiady LM. Decision Level Fusion of Iris and Signature Biometrics for Personal Identification using Ant Colony Optimization. Int J Eng Innovat Technol 2014; 3: 35-42.

14. Kaur S, Sharma P. Analysis of Multimodal Biometrics by Feature Level Fusion: A Review. Int J Adv Res Comput Sci Software Eng 2013; 3: 1388-1392.

15. Ross A, Govindarajan R. Feature Level Fusion Using Hand and Face Biometrics. Proc. of SPIE Conf. on Biometric Technol. for Human Identification II, Orlando, USA, 2005.

16. Akhtar Z, Fumera G, Marcialis GL, Roli F. Evaluation of serial and parallel multibiometric systems under spoofing attacks. Biometrics Theory, Applications and Systems (BTAS), IEEE Int. Conf. on Biometrics Compendium, Arlington, 2012, IEEE.

17. Yang J, Yang JY. Generalized K-L transform based combined feature extraction. Pattern Recogn Lett 2002; 35: 295-297.

18. Gangopadhyay A, Chatterjee O, Chatterjee A. Hand Shape Based Biometric Authentication System Using Radon Transform and Collaborative Representation Based Classification. Proc. of IEEE 2nd Int. Conf. on Image Inf. Process.(ICIIP- 2013), India, 2013, IEEE.

19. Jian-Da W, Siou-Huan Ye. Driver identification using finger-vein patterns with Radon transform and neural network. Expert Syst Appl 2009; 36: 5793-5799.

20. Huang NE, Shen Z, Long SR, Wu MC, Shih HH, Zheng Q, Yen NC, Tung CC, Liu HH. The empirical mode decomposition and the Hilbert spectrum for nonlinear and non-stationary time series analysis. Math Physical Eng Sci 1998; 454: 903-995. 
21. Rehman NU, Mandic DP. Empirical Mode Decomposition for Trivariate Signals. IEEE Trans Signal Process 2010; 58: 1059-1068.

22. Wang Y, Ruan Q, Pan X. Palm print recognition method using Dual-Tree complex wavelet transform and local binary pattern histogram. Proc Int Symp Intell Signal Process Comm Systems (ISPACS), Xiamen, 2007, IEEE.

23. He S, Wu QH, Saunders JR. Group Search Optimizer: An Optimization Algorithm Inspired by Animal Searching Behavior. IEEE Trans Evolutionary Computation, 2009, 13: 973-990.

24. Badawi AM. 'Hand vein database', at systems and biomedical engineering, Cairo University, Cairo, Egypt, 2005.

25. http://biometrics.put.poznan.pl/vein-dataset

26. http: //mla.sdu.edu.cn/sdumla-hmt.html

27. Conti V, Militello C, Sorbello F, Vitabile S. A frequencybased approach for features fusion in fingerprint and iris multimodal biometric identification systems. IEEE Trans Syst Man Cybernetics 2010; 40: 384-395.

28. Khalifa AB, Amara NEB. Bimodal biometric verification with different fusion levels. Proc 6th Int Multi-Conf Syst Signals Devices SSD Djerba IEEE 2009.

29. Lin CL, Fan KC. Biometric Verification Using Thermal Images of Palm-Dorsa Vein Patterns. IEEE Trans Circuits Syst Video Technol 2004; 14: 199-213.
30. Amayeh G, Bebis G, Erol A, Nicolescu M. Hand-based verification and identification using palm- finger segmentation and fusion. Computer Vision Image Understanding 2009; 113: 477-501.

31. Kumar A, Prathyusha KV. Personal authentication using hand vein triangulation and knuckle shape. IEEE Trans Image Process 2009; 18: 2127-2136.

32. Raghavendra R, Imran M, Rao A, Kumar GH. Multi modal biometrics: analysis of hand vein and palm print combination used for personal verification. Proc IEEE Int Conf Emerging Trends Eng Technol (ICETET) Goa IEEE 2010.

33. Ferrer MA, Morales A, Travieso CM, Alonso JB. Combining hand biometric traits for personal identification. Proc IEEE Int Carnahan Conf Secur Technol Zurich IEEE 2009.

\section{*Correspondence to}

Bharathi Subramaniam

Department of Electronics and Communication Engineering

Dr. Mahalingam College of Engineering and Technology

Tamil Nadu

India 